\title{
Measurement of the Dynamical Dipolar Coupling in a Pair of Magnetic Nanodisks Using a Ferromagnetic Resonance Force Microscope
}

\author{
B. Pigeau, ${ }^{*}$ C. Hahn, G. de Loubens, V. V. Naletov, ${ }^{\dagger}$ and O. Klein \\ Service de Physique de l'État Condensé (CNRS URA 2464), CEA Saclay, 91191 Gif-sur-Yvette, France \\ K. Mitsuzuka, D. Lacour, M. Hehn, S. Andrieu, and F. Montaigne \\ Institut Jean Lamour, UMR CNRS 7198, Université de Lorraine, 54506 Nancy, France
}

(Received 20 July 2012; published 10 December 2012)

\begin{abstract}
We perform a spectroscopic study of the collective spin-wave dynamics occurring in a pair of magnetic nanodisks coupled by the magnetodipolar interaction. We take advantage of the stray field gradient produced by the magnetic tip of a ferromagnetic resonance force microscope to continuously tune and detune the relative resonance frequencies between two adjacent nano-objects. This reveals the anticrossing and hybridization of the spin-wave modes in the pair. At the exact tuning, the measured frequency splitting between the binding and antibinding modes corresponds to the strength of the dynamical dipolar coupling $\Omega$. This accurate ferromagnetic resonance force microscope determination of $\Omega$ is measured versus the separation between the nanodisks. It agrees quantitatively with calculations of the expected dynamical magnetodipolar interaction in our sample.
\end{abstract}

DOI: 10.1103/PhysRevLett.109.247602

PACS numbers: 76.50.+g, 75.30.Ds, 85.75.- $-\mathrm{d}$

Studies of the collective dynamics in magnetic nanoobjects coupled by the dipolar interaction has recently attracted a lot of attention [1-9] due to its potential for creating novel properties and functionalities for information technology. It affects the writing time of closely packed storage media [10], the synchronization of spin transfer nano-oscillators [11], and more broadly the field of magnonics [12], which aims at using spin waves (SW) for information processes [13]. Despite the generic nature of the dynamic magnetodipolar interaction, its direct measurement has been elusive because it is difficult to reach a regime where this coupling is dominant. It requires that the strength of the dynamical dipolar coupling $\Omega$ exceeds both the deviation range of eigenfrequencies between coupled objects and the resonance linewidth. Large $\Omega$ are usually obtained by fabricating thick nano-objects having large magnetization and placed nearby. But the constraint of fabricating two nano-objects, whose SW modes both resonate within $\Omega$, is difficult to meet. For long wavelengths, the SW eigenfrequency is indeed very sensitive to imperfections in the confinement geometry. Moreover, a direct determination of the coupling strength between any two systems requires the ability to tune and detune them at least on the $\Omega$ range [14]. So far, the absence of a knob to do so with the individual frequencies of nearby magnetic objects has prevented a reliable measurement of $\Omega$.

Published by the American Physical Society under the terms of the Creative Commons Attribution 3.0 License. Further distribution of this work must maintain attribution to the author(s) and the published article's title, journal citation, and DOI.
In this Letter we demonstrate that ferromagnetic resonance force microscopy (f-MRFM) allows this quantitative measurement of $\Omega$. We rely on the field gradient of the magnetic tip as a means to fully tune and detune the resonance frequencies of two nanodisks by continuously moving the tip laterally above the pair of disks. At the position where the stray field of the tip compensates the deviation of internal field due to the patterning process, the splitting between the eigenfrequencies of the binding and antibinding modes is exactly equal to $\Omega$. By studying the coupling versus the detuning and the separation between the nanodisks, we shall demonstrate that f-MRFM provides a reliable means to measure $\Omega$, the mode hybridization, and its effect on the mode linewidth.

The magnetic material used for this study is a $t=26.7 \mathrm{~nm}$ thick Fe-V $(10 \% \mathrm{~V})$ film grown by molecular beam epitaxy on $\mathrm{MgO}(001)[15,16]$. This is a ferromagnetic alloy with very high magnetization, $4 \pi M_{s}=1.7 \times 10^{4} \mathrm{G}$, and very low magnetic Gilbert damping, $\alpha=2 \times 10^{-3}$. The film is patterned into disks by $e$-beam lithography and ion milling techniques. The geometrical pattern [Fig. 1(a)] consists in three pairs of nearby disks having the same nominal diameter $2 R=600 \mathrm{~nm}$ but different edge to edge separation: $s=$ 200, 400, and $800 \mathrm{~nm}$. Each set is separated by $3 \mu \mathrm{m}$ in order to avoid cross coupling. An isolated disk of identical diameter is also patterned for reference purpose. The sample is placed in the room temperature bore of an axial superconducting magnet. The disks are perpendicularly magnetized ( $z$ axis) by an external field of $1.72 \mathrm{~T}$ [17], sufficient to saturate all the disks. A linearly polarized microwave field $h_{\mathrm{rf}}$ is produced by a broadband Au strip-line antenna of width $5 \mu \mathrm{m}$ deposited on top of a $50 \mathrm{~nm}$ thick $\mathrm{Si}_{3} \mathrm{O}_{2}$ isolating layer, above the magnetic disks. 


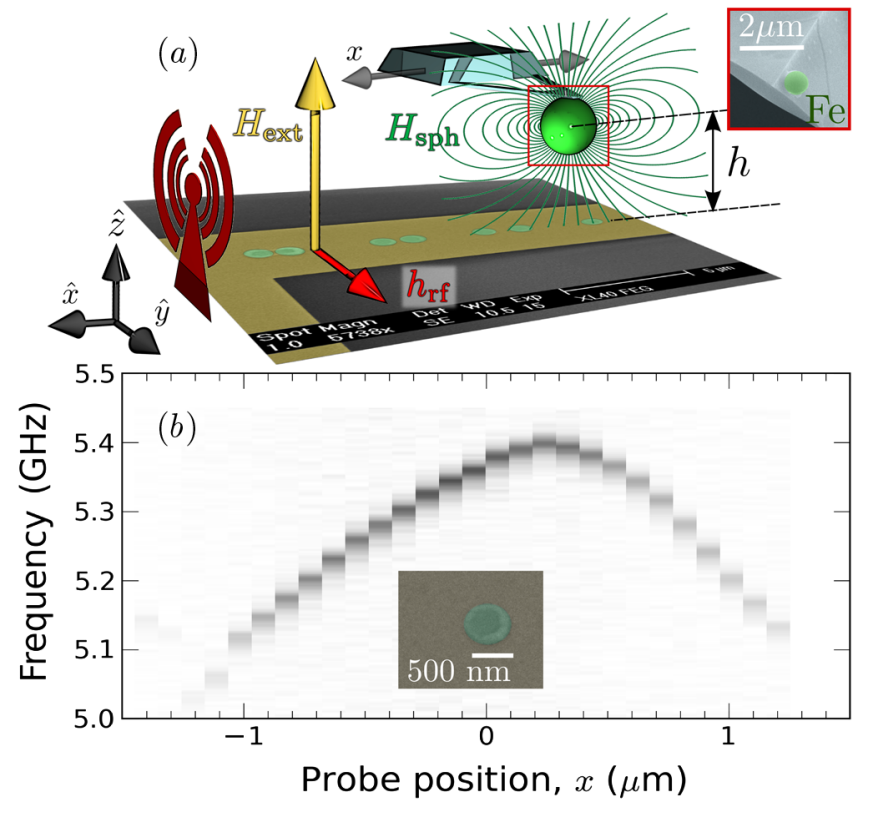

FIG. 1 (color online). (a) Schematic of the f-MRFM setup. An Fe sphere glued at the apex of a soft cantilever is scanned laterally above different pairs of Fe- $\mathrm{V}$ disks excited by a microwave field. Inset: SEM image of the tip. (b) Density plot of the f-MRFM signal versus the displacement $x$ of the sphere above an isolated disk. Inset: SEM image of the $2 R=600 \mathrm{~nm} \mathrm{Fe}-\mathrm{V}$ disk placed below the microwave antenna.

The FM RFM experiment is a novel technique that combines aspects of magnetic force microscopy and ferromagnetic resonance (FMR) to obtain a highly sensitive local probe of the magnetization dynamics $[19,20]$. It consists in mechanically detecting the diminution $\Delta M_{z}$ of spontaneous magnetization in the $\mathrm{Fe}-\mathrm{V}$ nanodisks associated with the Larmor precession [18]. The mechanical probe is a Biolever cantilever with an Fe nanosphere glued at its apex [see microscopy image in the inset of Fig. 1(a)]. The tip magnetization is characterized by cantilever magnetometry, relying on a reference coil to produce a calibrated field gradient at the tip location. Magnetometry as a function of the applied field shows a saturation of the tip moment at the value $m_{\text {sph }}=4 \times 10^{-10}$ emu once the applied field exceeds $0.65 \mathrm{~T}$. For calculation at a bias field of $1.72 \mathrm{~T}$, the tip stray field is therefore well approximated by $\boldsymbol{H}_{\mathrm{sph}}=-\boldsymbol{\nabla}\left(\boldsymbol{m}_{\mathrm{sph}} \cdot \boldsymbol{r} / r^{3}\right)$, the dipolar field created by a punctual magnetic moment placed at the center of the sphere. The role of the magnetic tip in f-MRFM is to create a field gradient tensor $\hat{\boldsymbol{G}}=\nabla \boldsymbol{H}_{\mathrm{sph}}$ on the sample in order to spatially code the resonance frequency and to provide a local detection [21].

These two features are illustrated by Fig. 1(b), which shows the dependence of the f-MRFM signal measured above the isolated disk versus the position of the tip on the $x$ axis. It displays the behavior of the lowest energy SW mode, where all spins are precessing in phase at the Larmor frequency. The cantilever is scanned at constant height $h$ above the sample surface. The position $x=0$ corresponds to placing the probe on the axis of the disk.

We first concentrate on the variation of the FMR resonance frequency versus the $x$ position of the sphere. It displays a bell curve due to the additional bias field produced by the tip [22]

$$
\omega(x)=\omega_{\mathrm{FMR}}+\gamma\left\{H_{\mathrm{sph}, z}(x)\right\},
$$

where the first term is the resonance frequency in the absence of the sphere and the second term is the gyromagnetic ratio $\gamma$ times the spatial average of the $z$ component of the stray field of the sphere over the disk volume. The curly bracket indicates that this average should be weighted by the spatial profile of the lowest energy SW mode [23]. The maximum shift of frequency occurs close to $x=0$, where the additional field from the f-MRFM sphere is maximal [24]. The slope of the wings is proportional to the lateral field gradient $G_{z x}$. For $h \gg 2 R$, it is maximum at $x \simeq 0.39 h$, where $h$ is the height between the sample surface and the sphere center. At this location, the gradient is about $G_{z x} \approx 2.7 m_{\mathrm{sph}} / h^{4}$. Since it is important to keep $h$ as large as possible for stability purposes, the optimal $h$ is reached when $\gamma G_{z x} R>\Omega$. For our settings, this occurs at $h=1.8 \mu \mathrm{m}$, leading to slope of about $0.3 \mathrm{GHz} / \mu \mathrm{m}$. At this distance, the maximum stray field of the sphere is about $140 \mathrm{G}$.

We then turn to the variation of the amplitude of the f-MRFM signal as a function of the position of the sphere in Fig. 1(b). The vertical force acting on the cantilever is $F_{z}=G_{z z} \Delta M_{z}$ [18]. The gradient $G_{z z}$ decays as the power $1 / x^{5}$, for large lateral displacement $x$. This decay ensures a local detection. Experimentally, the signal decreases by 1 order of magnitude when the probe is displaced by $1.2 \mu \mathrm{m}$ laterally.

We now discuss the same experiment above the pair of two $600 \mathrm{~nm}$ disks separated by $s=200 \mathrm{~nm}$. The result is displayed in Fig. 2(a). Here, $x=0$ corresponds to the middle of the pair. At each position $x$ of the f-MRFM probe, we can see two modes. The upper branch has two frequency maxima at $x_{1,2}=\mp 400 \mathrm{~nm}$, whose separation corresponds to the center to center distance between disk 1 and disk 2. The two maxima occur at slightly different frequencies, presumably due to a small difference in diameter between the two disks. When the probe is placed in between, $x_{1}<x<x_{2}$, the two levels anticross, which is a characteristic behavior of a coupled dynamic. Defining $\omega_{1,2}$ as the frequencies of the two uncoupled disks, the collective frequencies follow:

$$
\omega_{A, B}=\frac{\omega_{1}+\omega_{2}}{2} \pm \sqrt{\left(\frac{\omega_{1}-\omega_{2}}{2}\right)^{2}+\left(\frac{\Omega}{2}\right)^{2}},
$$

with $\Omega$ being the dynamical coupling strength. The two coupled eigenfrequencies $\omega_{A, B}$ correspond, respectively, to 

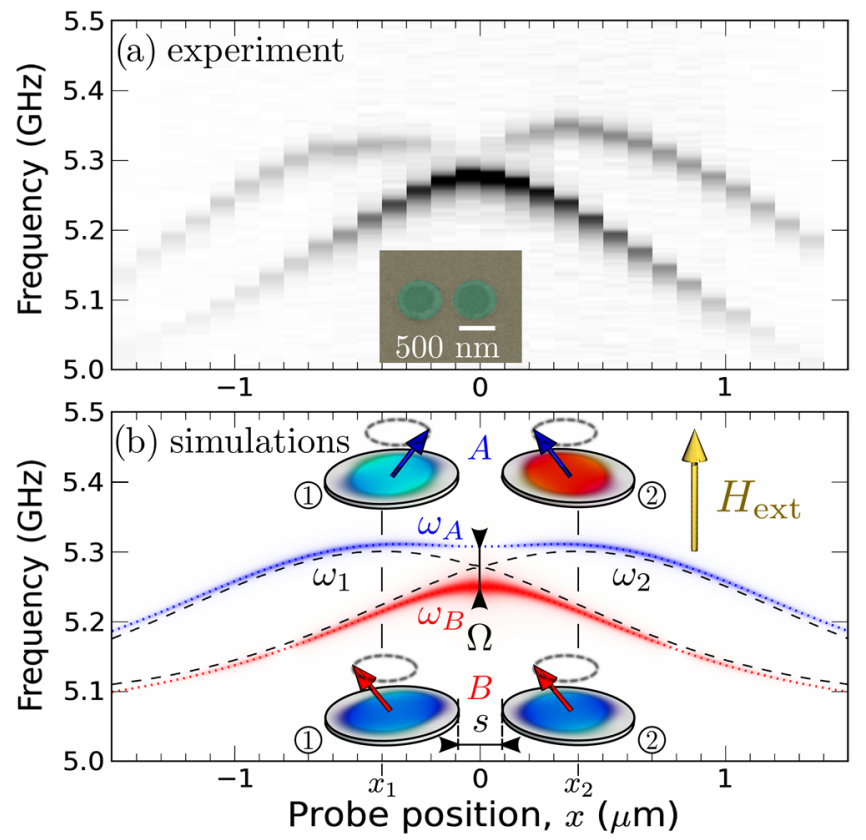

FIG. 2 (color online). (a) Density plot of the experimental f-MRFM spectra versus the displacement $x$ of the sphere above a pair of $600 \mathrm{~nm} \mathrm{Fe}-\mathrm{V}$ disks separated by $s=200 \mathrm{~nm}$. (b) Predicted behavior by micromagnetic simulations. The upper mode (blue) corresponds to the antibinding mode $(A)$, while the lower (red) shows the binding mode $(B)$. Insets: Simulated precession profiles in each disk for modes $(A)$ and $(B)$ at the anticrossing. The dashed lines would be the individual modes of each disk in the absence of dynamical coupling.

the antibinding mode $(A)$, where spins are precessing out of phase between the two disks, and to the binding mode $(B)$, where spins are precessing in phase [25]. In our f-MRFM experiment, $\omega_{1,2}$ both depend on $x$, see Eq. (1): $\omega_{1,2}(x)=\omega_{\mathrm{FMR}}+\gamma\left\{H_{\mathrm{sph}, z}\left(x-x_{1,2}\right)\right\}$. Using these dependencies in Eq. (2), one can obtain an analytical expression for the frequency difference $\omega_{A}(x)-\omega_{B}(x)$ observed in Fig. 2. At $x=0$, when $\omega_{1}=\omega_{2}$, the splitting $\omega_{A}-\omega_{B}$ is exactly $\Omega$. Using this analytical expression for the spatial dependence of the splitting, we have fitted $\Omega / 2 \pi=50 \pm$ $5 \mathrm{MHz}$. We emphasize that this splitting is 2.5 times larger than the linewidth, found to be $20 \mathrm{MHz}$. Theoretically, the coupling $\Omega$ for the magnetodipolar interaction is defined by [25]

$$
\Omega^{2}=4 \gamma^{2} h_{1,2} h_{2,1}
$$

$h_{i, j}$ represents the cross depolarization field produced by the $\mathrm{SW}$ in the $j$ th disk on the $i$ th disk $(i, j=1,2)$ [26-28]. It can be expressed as a function of the cross depolarization tensor elements, which have an analytical expression in the approximation of uniform precession [29]: $h_{i, j}=2 \pi M_{s}\left(\left\{N_{x x}^{i, j}\right\}+\right.$ $\left.\left\{N_{y y}^{i, j}\right\}\right)$. This formula reflects that the magnetodipolar interaction is anisotropic and, thus, it induces an elliptical precession in the two disks. For the separation $s=200 \mathrm{~nm}$, a numerical application yields $\left\{N_{x x}^{1,2}\right\} \approx-2\left\{N_{y y}^{1,2}\right\} \approx 0.0012$, which corresponds to a coupling field of about $10 \mathrm{G}$ between the two disks, or a coupling frequency $\Omega / 2 \pi=56 \mathrm{MHz}$, in good agreement with the measured value.

Another striking feature in Fig. 2(a) is the strong variation of the signal amplitude near the optimum coupling. We have plotted in Fig. 3(a) the amplitude of the f-MRFM signal versus the lateral displacement $x$ of the probe, showing both the near extinction of the antibinding mode $(A)$ and the strong enhancement of the binding mode $(B)$ near $x=0$. The ratio of hybridization in the coupled disks follows the expression:

$$
\left.\frac{c_{1}}{c_{2}}\right|_{A, B}=\left(\frac{\left(\omega_{1}-\omega_{2}\right) \mp \sqrt{\left(\omega_{1}-\omega_{2}\right)^{2}+\Omega^{2}}}{\Omega}\right)^{\mp 1} .
$$

Introducing the spatial dependence of $\omega_{1,2}$ described by Eq. (1) in Eq. (4), we can calculate the total force $F_{z} \propto$ $P\left[c_{1}^{2} G_{z z}\left(x-x_{1}\right)+c_{2}^{2} G_{z z}\left(x-x_{2}\right)\right]$ acting on the cantilever. The power efficiency $P=\left|c_{1}+c_{2}\right|^{2} h_{\mathrm{rf}}^{2}$ is proportional to the overlap integral between the uniform rf field and the collective SW mode (the vector sum of the transverse magnetization in the two disks) [25]. The predicted behavior of Eq. (4) is shown in Fig. 3(a) as the continuous lines. The difference between the two curves comes mainly from selection rules expressed in $P$. At $x=0$ (when $\omega_{1}$ and $\omega_{2}$ cross), the antibinding mode $(A)$ in Eq. (4) has $c_{1}=-c_{2}$, i.e., a precession with equal hybridization weight between the two disks and out of phase. The overlap with the
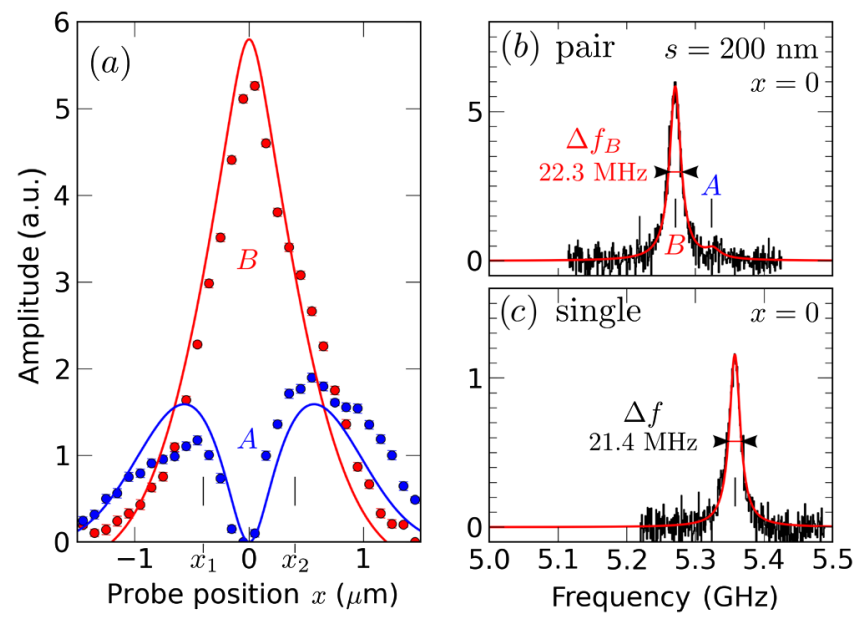

FIG. 3 (color online). (a) Variation of the amplitude in arbitrary units of the binding $(B$, red) and antibinding ( $A$, blue) resonances versus the lateral position of the probe for the two disks separated by $200 \mathrm{~nm}$. The solid lines correspond to the behavior following from Eq. (4) (see text). (b) Linewidth of the binding mode for the same pair at the tuning position $(x=0)$. (c) Linewidth of the single disk. Solid red lines are fit by Lorentzian curves. 
uniform rf field excitation is zero leading to a vanishing amplitude. In contrast, the binding mode $(B)$ has $c_{1}=+c_{2}$ at the anticrossing, i.e., a precession with equal hybridization weight, too, but in phase. It represents an enhancement of the absorbed power by a factor of $2^{2}$ compared to the amplitude in one disk.

We then study the effect of the magnetodipolar coupling on the linewidth of the collective mode. We observe that the linewidth does not change much with tuning and the overall variation with $x$ is below $5 \%$. At the optimal tuning $x=0$, the linewidth measured is $\Delta f=22.3 \pm 0.5 \mathrm{MHz}$ [see Fig. 3(b)] and it becomes slightly larger $\Delta f=23.1 \pm$ $0.5 \mathrm{MHz}$ at the maximum detuning $x=x_{1,2}$. For comparison we have displayed in Fig. 3(c) the linewidth observed above the single disk, $\Delta f=21.4 \pm 0.5 \mathrm{MHz}$. A small increase of the ratio $\Delta f / f$ is indeed expected for the dynamically coupled modes. This comes from the fact that this ratio is equal to $\Delta f / f=\alpha\left(H_{x}+H_{y}\right) / \sqrt{H_{x} H_{y}}$, where $\alpha$ is the Gilbert damping, and $H_{x}$ and $H_{y}$ represent the two stiffness fields which characterize the torque exerted on the magnetization when it is tipped along the $x$ or $y$ axis [30]. While the two stiffnesses are equal for the isolated disk, for the pair, the degree of hybridization as well as the nature of the mode ( $A$ or $B$ ) change the values and signs of $H_{x}$ and $H_{y}$. For the binding mode, the magneto-dipolar coupling generates an elliptical precession whose long axis is along the $x$-axis. The induced ellipticity $\mathcal{E}=\frac{\beta-1}{\beta+1} \frac{\Omega}{\omega_{B}}$ is maximum at the anticrossing $(x=0)$, reaching an amplitude of about $3 \%$ where $\beta=\left\{N_{x x}^{1,2}\right\} /\left\{N_{y y}^{1,2}\right\} \approx$ -2 . An increase of ellipticity induces an increase of the linewidth, a behavior consistent with the small additional broadening measured in our experiment.

The analytical model used above to analyze the data assumes a uniform magnetization throughout the magnetic body. To take more precisely into account the 3D texture of the magnetization and the static deformation induced by the probe, we have also calculated the eigenfrequencies versus $x$ using SPINFLOW3D, a finite element solver developed by In Silicio [31]. The disks are discretized with a mesh size of $10 \mathrm{~nm}$. At each position of the probe, we first calculate the equilibrium configuration in the disks. The Arnoldi algorithm is then used to compute the lowest eigenvalues of the problem as well as the associated eigenvectors. The result is represented in red and blue in Fig. 2(b) for the two lowest energy modes. The precession patterns associated with each mode at the anticrossing are shown in the inset. In this color representation, the hue indicates the phase (or direction) of the oscillating magnetization, while the brightness indicates its amplitude. The simulation results confirm very nicely the interpretation made above in terms of amplitude and peak position.

We have then repeated the same procedure on the two other pairs of disks, with larger edge to edge separation $s$. The strength of the dynamical coupling measured by f-MRFM is plotted versus $s$ in Fig. 4. The main result is

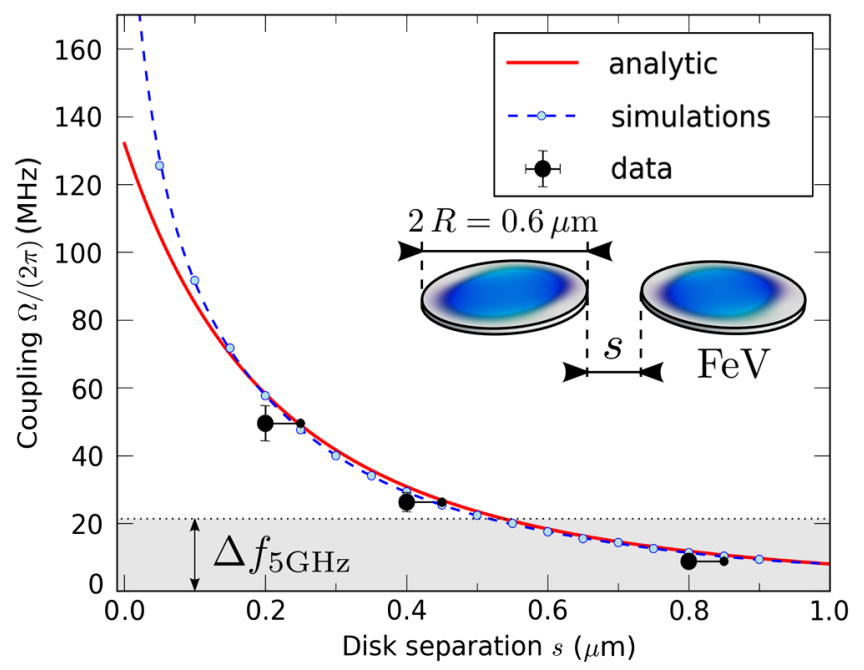

FIG. 4 (color online). Coupling strength versus the separation $s$ between two disks. The plot compares the experimental findings to the predicted amplitude of the magnetodipolar interaction either analytically (continuous line) or by micromagnetic simulations (small dots, dashed line is a guide to the eye).

that, with our experimental parameters, $s$ needs to be less than the diameter of the disks in order to have $\Omega /(2 \pi)$ larger than the linewidth $\Delta f$. Because of the geometrical origin of the dipolar coupling, meeting this criterion would require a smaller value of $s / R$ if the aspect ratio $t / R$ of the disks would decrease ( $t$ being the thickness). The data are plotted along with the analytical prediction (continuous line) and the simulations (dashed line with small dots). We observe an excellent overall agreement between the three sets of results, which exhibit a similar decay with $s$ (not a simple polynomial law [32]). Still, the experimental points are systematically slightly below the theoretical expectation. This could be explained by the fact that the disks are slightly smaller than their nominal value (e.g., due to some oxidation at their periphery), or that the true separation between the disks is slightly larger than expected, which we have represented on the graph by the horizontal error bars. The agreement between the analytical model and the simulation is very good until $s=0.1 \mu \mathrm{m}$. The discrepancy for very small $s$ is due to significative changes in the dynamic texture, which are not taken into account by the analytical model.

In conclusion, we have shown that f-MRFM enables a detailed investigation of the dynamical dipolar coupling between two nearby magnetic objects, owing to the possibility of the technique to study both the tuned and detuned regime on the same object. It has been applied to study the collective SW dynamics in pairs of nanodisks of Fe-V. Several signatures of the collective behavior have been experimentally evidenced and quantitatively explained: the anticrossing, the hybridization of the modes, and the effects on the linewidth. We believe that we have found a general method of characterization of the dynamic dipolar coupling between two nano-objects that will be very useful 
for the study of other more complex cases, in particular, in the field of magnonics.

This research work was partially supported by the French Grants VOICE ANR-09-NANO-006-01 and MARVEL ANR-2010-JCJC-0410-01.

*Corresponding author. benjamin.pigeau@cea.fr

†Present address: Physics Department, Kazan Federal University, Kazan 420008, Russian Federation.

[1] G. N. Kakazei, Y. G. Pogorelov, M. D. Costa, T. Mewes, P.E. Wigen, P.C. Hammel, V.O. Golub, T. Okuno, and V. Novosad, Phys. Rev. B 74, 060406 (2006).

[2] K. W. Chou, A. Puzic, H. Stoll, G. Schütz, B. V. Waeyenberge, T. Tyliszczak, K. Rott, G. Reiss, H. Brückl, I. Neudecker, D. Weiss, and C. H. Back, J. Appl. Phys. 99, 08F305 (2006).

[3] G. de Loubens, V. V. Naletov, M. Viret, O. Klein, H. Hurdequint, J. B. Youssef, F. Boust, and N. Vukadinovic, J. Appl. Phys. 101, 09F514 (2007)

[4] G. Gubbiotti, M. Madami, S. Tacchi, G. Carlotti, H. Tanigawa, and T. Ono, J. Phys. D 41, 134023 (2008).

[5] A. A. Awad, G. R. Aranda, D. Dieleman, K. Y. Guslienko, G. N. Kakazei, B. A. Ivanov, and F. G. Aliev, Appl. Phys. Lett. 97, 132501 (2010).

[6] H. Jung, Y.-S. Yu, K.-S. Lee, M.-Y. Im, P. Fischer, L. Bocklage, A. Vogel, M. Bolte, G. Meier, and S.-K. Kim, Appl. Phys. Lett. 97, 222502 (2010).

[7] A. Vogel, A. Drews, T. Kamionka, M. Bolte, and G. Meier, Phys. Rev. Lett. 105, 037201 (2010).

[8] S. Sugimoto, Y. Fukuma, S. Kasai, T. Kimura, A. Barman, and Y. C. Otani, Phys. Rev. Lett. 106, 197203 (2011).

[9] H. Ulrichs, V. E. Demidov, S. O. Demokritov, A. V. Ognev, M. E. Stebliy, L.A. Chebotkevich, and A. S. Samardak, Phys. Rev. B 83, 184403 (2011).

[10] V. V. Kruglyak, P. S. Keatley, A. Neudert, R. J. Hicken, J. R. Childress, and J.A. Katine, Phys. Rev. Lett. 104, 027201 (2010).

[11] A. D. Belanovsky, N. Locatelli, P. N. Skirdkov, F. A. Araujo, J. Grollier, K. A. Zvezdin, V. Cros, and A. K. Zvezdin, Phys. Rev. B 85, 100409 (2012).

[12] V. V. Kruglyak, S. O. Demokritov, and D. Grundler, J. Phys. D 43, 264001 (2010).

[13] A. D. Karenowska, J. F. Gregg, V.S. Tiberkevich, A. N. Slavin, A. V. Chumak, A. A. Serga, and B. Hillebrands, Phys. Rev. Lett. 108, 015505 (2012).

[14] Y. Kubo, C. Grezes, A. Dewes, T. Umeda, J. Isoya, H. Sumiya, N. Morishita, H. Abe, S. Onoda, T. Ohshima, V. Jacques, A. Dréau, J.-F. Roch, I. Diniz, A. Auffeves, D. Vion, D. Esteve, and P. Bertet, Phys. Rev. Lett. 107, 220501 (2011).
[15] F. Bonell, S. Andrieu, F. Bertran, P. Lefevre, A. Ibrahimi, E. Snoeck, C.-V. Tiusan, and F. Montaigne, IEEE Trans. Magn. 45, 3467 (2009).

[16] K. Mitsuzuka, D. Lacour, M. Hehn, S. Andrieu, and F. Montaigne, Appl. Phys. Lett. 100, 192406 (2012).

[17] In fact, the external field is tilted by $\theta_{H} \simeq 2^{\circ}$ in the $x$ direction. Our setup does not allow in situ adjustment of this small misalignment. Although it can be easily integrated in a complete analysis [18], this point will be neglected for the sake of simplicity, as it brings minor correction.

[18] O. Klein, G. de Loubens, V. V. Naletov, F. Boust, T. Guillet, H. Hurdequint, A. Leksikov, A. N. Slavin, V. S. Tiberkevich, and N. Vukadinovic, Phys. Rev. B 78, 144410 (2008).

[19] D. Rugar, C. S. Yannoni, and J. A. Sidles, Nature (London) 360, 563 (1992).

[20] Z. Zhang, P. C. Hammel, and P.E. Wigen, Appl. Phys. Lett. 68, 2005 (1996).

[21] I. Lee, Y. Obukhov, G. Xiang, A. Hauser, F. Yang, P. Banerjee, D. Pelekhov, and P. Hammel, Nature (London) 466, 845 (2010).

[22] Y. Obukhov, D. V. Pelekhov, J. Kim, P. Banerjee, I. Martin, E. Nazaretski, R. Movshovich, S. An, T. J. Gramila, S. Batra, and P. C. Hammel, Phys. Rev. Lett. 100, 197601 (2008).

[23] $\{\boldsymbol{H} \operatorname{sph}\}=\int_{S} \frac{J_{0}\left(\kappa_{0,0} \sqrt{x^{2}+y^{2}} / R\right)^{2} \boldsymbol{H} \operatorname{sph}(x, y)}{\pi J_{1}\left(\kappa_{0,0}\right)^{2} R^{2}} d x d y$ where $S$ is the area of the disk, $J_{\ell}$ is the $\ell$ th order Bessel function, and $\kappa_{\ell, n}$ is its $n$th order root [18].

[24] The asymmetries of the bell-shape curve (maximum of frequency shifted with respect to $x=0$ and to the maximum of amplitude, and different slopes in the wings) are due to the misalignment $\theta_{H}$ noted in Ref. [16].

[25] V. V. Naletov, G. de Loubens, G. Albuquerque, S. Borlenghi, V. Cros, G. Faini, J. Grollier, H. Hurdequint, N. Locatelli, B. Pigeau, A. N. Slavin, V.S. Tiberkevich, C. Ulysse, T. Valet, and O. Klein, Phys. Rev. B 84, 224423 (2011).

[26] M. P. Kostylev, A. A. Stashkevich, N. A. Sergeeva, and Y. Roussigné, J. Magn. Magn. Mater. 278, 397 (2004).

[27] M. Beleggia, S. Tandon, Y. Zhu, and M. D. Graef, J. Magn. Magn. Mater. 278, 270 (2004).

[28] R. Verba, G. Melkov, V. Tiberkevich, and A. Slavin, Phys. Rev. B 85, 014427 (2012).

[29] S. Tandon, M. Beleggia, Y. Zhu, and M. De Graef, J. Magn. Magn. Mater. 271, 9 (2004).

[30] A. G. Gurevich and G. A. Melkov, Magnetization Oscillations and Waves (CRC Press, Boca Raton, FL, 1996).

[31] http://www.insilicio.fr/pdf/Spinflow_3D.pdf.

[32] O. V. Sukhostavets, J. M. Gonzalez, and K. Y. Guslienko, Appl. Phys. Express 4, 065003 (2011). 